Case Report

\title{
Anti-Ganglioside Antibody Negative Miller Fisher Syndrome with Atypical Features: A Case Report
}

\author{
Asif labal', Abhishek Yadav', Lalit Chandra Kummetha', Naresh Kumar ${ }^{3}$, Gaurav Sharma', \\ Princey Poonam', RS Ahlawat $^{4}$
}

${ }^{1}$ Resident, ${ }^{2}$ Senior Resident, Deapartment of Medicine, ${ }^{4}$ Director Professor, Maulana Azad Medical College, New Delhi, India. ${ }^{3}$ Professor of Medicine \& Head, Pulmonary Medicine, Maulana Azad Medical College, New Delhi, India.

DOI: https://doi.org/10.24321/2454.325X.202110

I $\mathbf{N} \mathbf{F} \mathbf{O}$

\section{Corresponding Author:}

Naresh Kumar, Pulmonary Medicine, Maulana Azad Medical College, New Delhi, India.

E-mail Id:

drnareshmamc@gmail.com

Orcid Id:

https://orcid.org/0000-0003-4581-609X

How to cite this article:

Iqbal A, Yadav A, Kummetha LC, Kumar N, Sharma G, Poonam P, Ahlawat RS. Anti-Ganglioside Antibody Negative Miller Fisher Syndrome with Atypical Features: A Case Report. Int J Preven Curat Comm Med. 2021;7(2):26-28.

Date of Submission: 2021-05-10

Date of Acceptance: 2021-06-21

\section{$\begin{array}{llllllll}\mathbf{A} & \mathbf{B} & \mathbf{S} & \mathbf{T} & \mathbf{R} & \mathbf{A} & \mathbf{C} & \mathbf{T}\end{array}$}

Miller Fisher Syndrome (MFS), a variant of Guillain Barre Syndrome (GBS), is an immune-mediated neuropathy presenting with the classical clinical triad of ophthalmoplegia, ataxia, and areflexia. Although the clinical triad is the cardinal diagnostic clue, it can also present with a variety of other atypical neurological symptoms and signs beyond the classical triad. IgG anti-GQ1b antibodies are a powerful serological marker for the diagnosis of MFS, however, they can be absent in 10$15 \%$ cases of MFS. Here, we are describing a case of a 55-year old lady with an anti-ganglioside negative case of MFS with ptosis and bulbar palsy, who improved with IVIg.

Keywords: Guillain Barre Syndrome (GBS), Miller Fisher Syndrome (MFS), Anti-Ganglioside Negative, Atypical Features, Ptosis, Bulbar Palsy

\section{Introduction}

The incidence of Guillain Barre Syndrome (GBS) is approximately 1-2 in 100,000 population with Miller Fisher syndrome (MFS) variant representing only a tiny subset of these cases. MFS represents only $15 \%$ to $25 \%$ of GBS in the Asian population and $5 \%$ in the Western population, thus making it a very rare condition. ${ }^{1}$ Atypical manifestations can present in $30 \%$ of patients. ${ }^{2}$ Anti-GQ1b antibodies are powerful serological markers in $90 \%$ of cases of MFS. ${ }^{3}$ Here, we report a case of anti-GQ1b negative MFS who presented with ptosis and bulbar palsy.

\section{Case History}

A 55-year-old lady presented to the emergency department with five days history of ptosis, diplopia, dysphagia with nasal regurgitation of food, and recurrent falls. She denied any diurnal variation of these symptoms, weakness of limbs, loss of consciousness, seizures, head trauma, recent vaccination, joint pain, or substance abuse. She had a history of upper respiratory tract infection twelve days back. The patient's past medical history consisted of hypothyroidism, rheumatic heart disease and severe mitral stenosis with atrial fibrillation.

At admission, the patient was conscious and oriented with Glasgow Coma Scale (GCS) score of 15/ 15. She was afebrile with an irregularly irregular pulse and heart rate of 96/ $\mathrm{min}$, blood pressure of $126 / 82 \mathrm{mmHg}$, and respiratory rate of $16 /$ min. Cranial nerves examination revealed bilateral ptosis with restricted ocular movements in all directions bilaterally. Pupils were equal-sized and normally reacting. 
Bilateral lower motor neuron type facial nerve palsy was present. Dysarthria was present with nasal intonation of voice and absent pharyngeal reflex. Motor examination revealed that the power was $5 / 5$ in all the four extremities with global areflexia and flexor plantar response bilaterally. Vibration and proprioception were impaired in all four limbs. Examination of the rest of the sensory system, cerebellar, and autonomic system was unremarkable. The patient was not able to stand due to severe ataxia.

At initial presentation, differential diagnosis of cardioembolic stroke involving brainstem, myasthenia gravis and botulism were kept. Acute onset cranial neuropathies without diurnal variation decreased the possibility of myasthenia gravis. We also considered the possibility of botulism but the probability was kept low due to the absence of pupillary palsy, autonomic involvement, and limb weakness. In the presence of the classical triad of acute onset external ophthalmoplegia with global areflexia and ataxia, the possibility of MFS was considered.

Laboratory investigations revealed normal haematological and metabolic parameters, thyroid function, and cardiac markers. Imaging studies including contrast-enhanced computed tomography of the chest and 2-dimensional echocardiography, magnetic resonance imaging (MRI) of the brain with angiography were normal. A lumbar puncture was performed that revealed albuminocytologic dissociation with normal cell counts and elevated protein of $128 \mathrm{mg} /$ dl. Nerve Conduction Studies (NCS) were done. Motor NCS showed prolonged distal latency in the right median nerve with normal CMAP and conduction velocities in all the recorded nerves. Right common peroneal and tibial nerves showed prolonged F waves. Sensory NCS showed non-recordable left median and ulnar nerves while the left sural nerve was spared. Serology for group A streptococcal antigen, chlamydia, Epstein Barr virus, HIV, hepatitis B, mycoplasma, and antinuclear antibody was negative. The electroencephalogram was normal. Electromyography could not be done. Serological testing for anti-ganglioside IgG against GQ1b, GM1, GM2, GM3, GD1a, GD1b, GT1b, and GQ1b was performed and revealed negative results.

Based on the clinical presentation, albuminocytological dissociation in CSF analysis, findings on NCS, and negative brain imaging diagnosis of MFS with atypical features were made. She was started on intravenous immunoglobulins (IVIG) $400 \mathrm{mg} / \mathrm{kg} /$ day for 5 days along with supportive care. Ptosis and other features started improving after 2 days of the start of therapy and there was no further clinical deterioration (Figure 1).

The patient was discharged on the 15th day of admission after significant clinical improvement in dysphagia, dysarthria, and ataxia. She was asymptomatic on follow up visits.

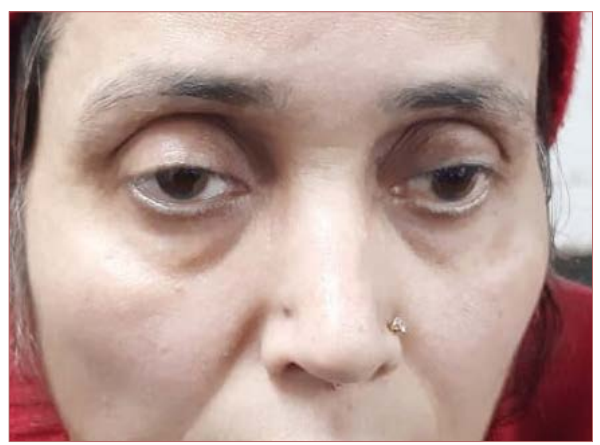

(a)

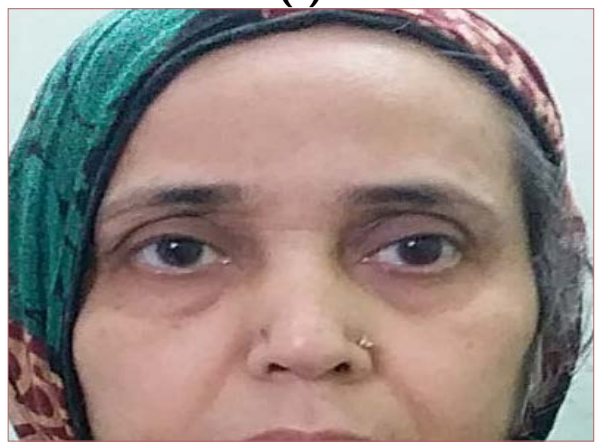

(b)

Figure I(a).Ptosis (Before Treatment on the Day of Admission) (b) Recovery of Ptosis (At Discharge on the I5th Day)

\section{Discussion}

MFS can present itself with a wide range of neurological manifestations. Pure MFS is described as the presence of only the triad of ophthalmoplegia, ataxia, and areflexia. However, the absence of any one of the components among the triad classifies it into incomplete MFS. ${ }^{4}$ Jung J et al. reported atypical clinical manifestations such as headache, facial palsy, taste impairment, ptosis, and divergence insufficiency in $30 \%$ of MFS. ${ }^{2}$ Mori et al. described 50 cases of MFS in which atypical clinical features such as blepharoptosis (58\%), pupillary palsies (42\%), facial palsy (32\%), bulbar palsy (26\%), limb dysesthesia (24\%), and motor weakness (20\%) were present, with or without the clinical triad. ${ }^{1}$ Our case presented with ptosis and bulbar palsy, atypical for MFS.

The role of anti-GQ1b ganglioside antibody in MFS was first described in 1992. ${ }^{5}$ Pathophysiology can be explained by molecular mimicry of GQ1b mimicking structure on the surface of infectious agents and of neural ganglioside antigens. The GQ1b epitope is strongly expressed in oculomotor nerves, dorsal root ganglion neurons, and muscle spindles, and these localisations explain the classic triad of symptoms seen in patients with MFS.

There is a scarcity of literature on the pathophysiology of MFS in anti-Gq1b negative cases. Some cases have been linked with antibodies against other gangliosides and ganglioside complexes (GSC) that can generate new 
epitopes. In an extensive 2-year study by Koga et al. on 207 MFS patients, $24(12 \%)$ anti-Gq1b seronegative patients were identified. They revealed that amongst anti-Gq1b seronegative patients, $17 \%$ had isolated IgG anti-ganglioside antibodies against other gangliosides (GM1b or GD1c), $17 \%$ had antibodies against ganglioside complexes (GT1a) GM1 and GQ1b/GM1), and 29\% had IgG antibodies against ganglioside-like lipo-oligosaccharide (LOS) of Campylobacter jejuni isolates. ${ }^{6}$ Recent studies also indicate the role of antiglutamic acid decarboxylase (GAD) antibodies in anti-GQ1b seronegative cases. ${ }^{7}$

Clinical presentations of GQ1b seronegative and seropositive patients are indistinguishable except for the male predominance and history of preceding gastrointestinal illness in seronegative patients. ${ }^{6}$ However, GQ1bseronegative, as well as GSC-seropositive patients, may show recurrence of the disease. ${ }^{8}$ Anti-ganglioside antibody profile including anti-GQ1b was negative in our case. We could not test her for GSC and anti-GAD due to financial constraints.

To conclude, MFS is a rare immune-mediated neuropathy with classical triad of ophthalmoplegia, ataxia, and areflexia. However, it can present with other atypical neurological symptoms as well. IgG anti-GQ1b antibodies may be absent on $10-15 \%$ cases of MFS. MFS responds favourably to IVIg when treated timely.

\section{Conflict of Interest: None}

\section{References}

1. Mori M, Kuwabara S, Fukutake T, Yuki N, Hattori T. Clinical features and prognosis of Miller Fisher syndrome. Neurol. 2001;56:1104-6. [PubMed] [Google Scholar]

2. Jung JH, Oh EH, Shin JH, Kim DS, Choi SY, Choi KD, Choi $\mathrm{JH}$. Atypical clinical manifestations of Miller Fisher syndrome. Neurol Sci. 2019;40:67-73. [PubMed] [Google Scholar]

3. Chiba A, Kusunoki S, Obata H, Machinami R, Kanazawa I. Serum anti-GQ1b IgG antibody is associated with ophthalmoplegia in Miller Fisher syndrome and GuillainBarré syndrome: clinical and immunohistochemical studies. Neurol. 1993;43:1911-7. [PubMed] [Google Scholar]

4. Wakerley BR, Uncini A, Yuki N; GBS Classification Group. Erratum: Guillain-Barré and Miller Fisher syndromes-new diagnostic classification. Nat Rev Neurol. 2014;10:537-44. [PubMed] [Google Scholar]

5. Chiba A, Kusunoki S, Shimizu T, Kanazawa I. Serum IgG antibody to ganglioside GQ1b is a possible marker of Miller Fisher syndrome. Ann Neurol. 1992;31:677-9. [PubMed] [Google Scholar]

6. Koga M, Gilbert M, Takahashi M, Li J, Hirata K, Kanda
T, Yuki N. GQ1b-seronegative Fisher syndrome: clinical features and new serological markers. J Neurol. 2012;259:1366-74. [PubMed] [Google Scholar]

7. Pietrini V, Pavesi G, Andreetta F. Miller Fisher syndrome with positivity of anti-GAD antibodies. Clin Neurol Neurosurg. 2013;115:1479-81. [PubMed] [Google Scholar]

8. Ito H, Hatanaka Y, Fukami Y, Harada Y, Kobayashi R, Okada H, Uchibori A, Chiba A, Okuda S. Anti-ganglioside complex antibody profiles in a recurrent complicated case of GQ1b-seronegative miller fisher syndrome and Bickerstaff brainstem encephalitis: a case report. BMC Neurol. 2018;18:72. [PubMed] [Google Scholar] 